Research Article

\title{
Intensity Distribution of Partially Coherent Array Finite Airy Beams Propagating in Atmospheric Turbulence
}

\author{
Long Jin (D), Zirui Zhang, Nuo Wang, Zixin Liu, Yuwei Deng, and Yanhua Fu \\ Department of Basic Science, Hubei University of Automotive Technology, 167 Checheng West Road, Shiyan, \\ Hubei 442002, China \\ Correspondence should be addressed to Long Jin; crazyjinlong@163.com
}

Received 26 October 2020; Revised 11 January 2021; Accepted 23 January 2021; Published 8 February 2021

Academic Editor: Stefan Wabnitz

Copyright $(92021$ Long Jin et al. This is an open access article distributed under the Creative Commons Attribution License, which permits unrestricted use, distribution, and reproduction in any medium, provided the original work is properly cited.

Based on the extended Huygens-Fresnel integral and the Rytov phase structure function, the closed-form expression of a partially coherent array finite Airy beams (PCAFABs) cutting through the Kolmogorov atmospheric turbulence is derived in the space domain under the paraxial approximation. The characteristics of the PCAFABs evoluting in the atmospheric environment are investigated in detail on the basis of the derived wave propagation formulae. We mainly illustrate the intensity profile of this beam changed with the truncation parameter, coherence length, and turbulence factor at several cross sections of the atmospheric space by means of numerical figures. It is convinced that the present concept and derived conclusions will provide useful exploration for learning the optical properties of the PCAFABs transmitting in the atmospheric turbulence, especially for free-space optical communication area.

\section{Introduction}

Atmospheric laser communication is featured with the advantages of large channel capacity, good confidentiality, convenient installation, and low production cost compared with other wireless and wired communication equipment [1]. However, the diffraction and non-self-heal effects of Gaussian beam are very obvious in the transmission process when it meets obstacles or blocks [2]. Moreover, it is difficult to overcome the defects such as low energy utilization rate, large spot divergence angle, slow transmission rate, and high bit error rate for Gaussian beam even with the adoption of partial coherence technology [3]. Therefore, it is becoming the research hot spot for science and engineering experts to find a kind of novel laser light source itself owning the ability to resist atmospheric turbulence in recent decades.

The Airy beam with finite energy was experimentally proposed by Siviloglou et al. in 2007, and they mainly used the computer-controlled liquid crystal spatial light modulator to generate the Airy beam model coupled with an exponential term [4]. From then on, it is believed that the self-accelerating, nondiffracting, and self-healing properties of the Airy beam will be of great value in long-range, high-speed atmospheric communication. In 2011, Chu examined the centroid position and skewness of Airy beam intensity in atmospheric turbulence [5]. In 2013, Eyyuboğlu et al. discussed the propagation characteristics of both Airy beam and partially coherent Airy beam in turbulent media, respectively [6, 7]. In 2014, Yang et al. studied the degree of polarization of vector partially coherent Airy beams propagating through atmospheric turbulence [8]. In 2019, Li et al. explored the intensity and phase distributions of a ring Airy Gaussian vortex beam propagating in atmospheric turbulence [9]. However, not only is the amount of energy carried by a single-beam source extremely limited, but also the asymmetric transverse intensity property of the single Airy beam is adverse in some optical application [10], so it is necessary to arrange the multiple Airy beams in the radial symmetry, which is called the array Airy beam [11]. In the past few years, research on the array Airy beam cultivated a new interdisciplinary area. In 2010, Gu et al. investigated the scintillation properties of Airy beam arrays in atmospheric turbulence, and they found that the scintillation of an Airy beam array is significantly reduced and close to the theoretical minimum [12]. In 2014, Chen et al. derived the 
average intensity of both phase-locked and non-phase-locked Airy array beams propagating through atmospheric turbulence, and they obtained that the differences between the propagation properties of phase-locked Airy array beams and those of non-phase-locked ones become unobservable when the atmospheric turbulence is strong enough [13]. In 2017, Lu et al. investigated the generation of coherent and incoherent Airy beam arrays and the comparisons of their scintillation characteristics in atmospheric turbulence, and they pointed out that the incoherent Airy beam arrays have a smaller scintillation index than the coherent one in the same turbulent condition due to the coherence reduction of the constituent beamlets [14]. In 2020, Suarez et al. presented the experimental generation and analysis of both the intensity and phase of an optical vortex beam originating from a superposition of Airy-vortex beams [15]. However, as far as we know, there are few studies on the evolution rules of the partially coherent array finite Airy beams (PCAFABs) in atmospheric turbulence. We are convinced that the rational use of the evolution properties of PCAFABs in atmospheric turbulence will provide a powerful and convenient way for free-space optical communication.

In this paper, we will research systematically the PCAFAB intensity outlines at some cross sections of the atmospheric environment, and the beam transverse self-acceleration parabolic trajectory with the changing of the transmission distance. The rest of the paper is organized as follows: in Section 2, the general intensity expression of the PCAFABs passing through the Kolmogorov atmospheric turbulence is derived in the space domain by the extended Huygens-Fresnel integral under the paraxial approximation. In Section 3, the intensity distribution of the PCAFABs changed with the truncation parameter, coherence length, and turbulence factor are explored by numerical examples in detail. In Section 4, a clear, concise summary is outlined at the end of our theses.

\section{Mathematical Model and Calculation Method}

The electric field of the two-dimensional single finite Airy beam at source plane $(z=0)$ can be described as [16]

$$
\begin{aligned}
E_{1}\left(r^{\prime}, z=0\right)= & E_{1}\left(x_{0}, 0\right) \cdot E_{1}\left(y_{0}, 0\right)=A_{i}\left(\frac{x_{0}}{\omega_{0}}\right), \\
& \times \exp \left(a_{0} \frac{x_{0}}{\omega_{0}}\right) \cdot A_{i}\left(\frac{y_{0}}{\omega_{0}}\right) \times \exp \left(a_{0} \frac{y_{0}}{\omega_{0}}\right),
\end{aligned}
$$

where the vector $r^{\prime}=\left(x_{0}, y_{0}\right)$ denotes the transverse coordinates at two axial directions, $\omega_{0}$ characters the incident beam waist size, $0<a_{0}<1$ provides the truncation parameter, and the symbol $A_{i}$ expresses the Airy function, of which the standard integral function is [17]

$$
A_{i}(x)=\frac{1}{2 \pi} \times \int_{-\infty}^{+\infty} \exp \left[i \frac{u^{3}}{3}+i u x\right] \mathrm{d} u
$$

The symmetric array finite Airy beams (AFABs) can be constituted through superimposing four parts of single Airy beams. By adjusting each beamlet's phase parameter, the incident AFABs are given by [18]

$$
\begin{aligned}
E_{2}\left(r^{\prime}, z=0\right)= & E_{1}\left(x_{0}, 0\right) \times E_{1}\left(y_{0}, 0\right) \\
& +E_{1}\left(-x_{0}, 0\right) E_{1}\left(y_{0}, 0\right)+E_{1}\left(x_{0}, 0\right) \\
& \times E_{1}\left(-y_{0}, 0\right)+E_{1}\left(-x_{0}, 0\right) \times E_{1}\left(-y_{0}, 0\right) .
\end{aligned}
$$

The cross-spectral density function (CSDF) is usually used to describe a partially coherent laser beam. Here, we select the Gaussian Schell model, leading to the expression of the incident PCAFABs as [19]

$$
W\left(r_{1}^{\prime}, r_{2}^{\prime}, 0\right)=E_{2}\left(r_{1}^{\prime}, 0\right) \times E_{2}^{*}\left(r_{2}^{\prime}, 0\right) \times \exp \left[-\frac{\left(r_{1}^{\prime}-r_{2}^{\prime}\right)^{2}}{\sigma^{2}}\right]
$$

where $\sigma$ represents the initial coherence length. The CSDF of PCAFABs evoluting in the atmospheric turbulence can be evaluated by the extended Huygens-Fresnel integral. Under the paraxial approximation, the integral expressions for this wave propagation at arbitrary $z$-plane are given by [20]

$$
\begin{aligned}
& W\left(r_{1}, r_{2}, z\right)=\left(\frac{k}{2 \pi z}\right)^{2} \int_{-\infty}^{+\infty} \int_{-\infty}^{+\infty} \int_{-\infty}^{+\infty} \int_{-\infty}^{+\infty} \\
& \cdot \exp \left\{\frac{i k}{2 z}\left[\left(r_{1}-r_{1}^{\prime}\right)^{2}-\left(r_{2}-r_{2}^{\prime}\right)^{2}\right]\right\} \times \\
& W\left(r_{1}^{\prime}, r_{2}^{\prime}, 0\right) \times<\exp \left[\varphi\left(r_{1}^{\prime}, r_{1}\right)+\varphi^{*}\left(r_{2}^{\prime}, r_{2}\right)\right] \\
&>_{m} \mathrm{~d} r_{1}^{\prime} \mathrm{d} r_{2}^{\prime},
\end{aligned}
$$

where $W\left(r_{1}, r_{2}, z\right)$ and $W\left(r_{1}^{\prime}, r_{2}^{\prime}, z\right)$ are the CDSF of PCAFABs at source and the output planes, respectively, $k=2 \pi / \lambda$ is the wave number with being the $\lambda$ wavelength, $r_{1}$ and $r_{2}$ are two different coordinate vectors, respectively, $z$ is the propagation distance, $\varphi(r, r)$ denotes the complex random phase fluctuation of wave transmission in atmospheric turbulence, and $\langle\dot{ }\rangle_{m}$ represents the ensemble average and can be written approximately by the Rytov phase structure function [21]: 


$$
\begin{aligned}
& <\exp \left[\varphi\left(r_{1}^{\prime}, r_{1}\right)+\varphi^{*}\left(r_{2}^{\prime}, r_{2}\right)\right]>_{m} \\
& =\exp \left[-\frac{\left.\left(r_{1}^{\prime}-r_{2}^{\prime}\right)^{2}+\left(r_{1}^{\prime}-r_{2}^{\prime}\right)\left(r_{1}-r_{2}\right)+\left(r_{1}-r_{2}\right)^{2}\right]=}{\rho_{0}^{2}}\right] \\
& \exp \left[-\frac{\left(x_{1}^{\prime}-x_{2}^{\prime}\right)^{2}+\left(y_{1}^{\prime}-y_{2}^{\prime}\right)^{2}}{\rho_{0}^{2}}\right] \\
& \quad \times \exp \left[-\frac{\left(x_{1}-x_{2}\right)^{2}+\left(y_{1}-y_{2}\right)^{2}}{\rho_{0}^{2}}\right] \times \\
& \exp \left[-\frac{\left(x_{1}^{\prime}-x_{2}^{\prime}\right)\left(x_{1}-x_{2}\right)+\left(y_{1}^{\prime}-y_{2}^{\prime}\right)\left(y_{1}-y_{2}\right)}{\rho_{0}^{2}}\right],
\end{aligned}
$$

where $\rho_{0}=\left(0.545 C_{n} 2_{k} 2_{z}\right)-3 / 5$ is known to be the coherence length and $\mathrm{Cn} 2$ is the structure constant of the refractive index fluctuations of the turbulence. Because the PCAFABs are symmetric in $x$ and $y$ directions, the $W(r 1, r 2$, z) can be separated with each other; that is to say,

$$
W\left(r_{1}, r_{2}, z\right)=W\left(x_{1}, x_{2}, z\right) \times W\left(y_{1}, y_{2}, z\right) .
$$

Demanding that $x_{1}=x_{2}=x, y_{1}=y_{2}=y$, the intensity expression for the partially coherent beam can be written by

$$
\begin{aligned}
& I(x, z)=W(x, x, z), \\
& I(y, z)=W(y, y, z) .
\end{aligned}
$$

Finally, the PCAFAB intensity distribution at the receiver plane is obtained as

$$
\begin{aligned}
I(r, z)= & I(x, z) \times I(y, z)+I(-x, z) \times I(y, z)+I(x, z) \\
& \times I(-y, z)+I(-x, z) \times I(-y, z),
\end{aligned}
$$

where

$$
\begin{aligned}
I(x, z)= & A_{i}\left\{\frac{x}{\omega_{0}}-\left[\frac{z^{2}}{4 k \omega_{0}^{4}}-\left(4 a_{0} b_{0}+b_{0}^{2}\right)\right]+\frac{z\left(a_{0}+b_{0}\right)}{k \omega_{0}^{2}} i\right\} \times \\
& A_{i}\left\{\frac{x}{\omega_{0}}-\left[\frac{z^{2}}{4 k \omega_{0}^{4}}-\left(4 a_{0} b_{0}+b_{0}^{2}\right)\right]-\frac{z\left(a_{0}+b_{0}\right)}{k \omega_{0}^{2}} i\right\} \times \\
& \exp \left\{2\left(a_{0}+b_{0}\right)\left\{\frac{x}{\omega_{0}}-\left[\frac{z^{2}}{2 k^{2} \omega_{0}^{4}}-\left(4 a_{0} b_{0}+b_{0}^{2}\right)\right]\right\}\right. \\
& \left.+\left(3 a_{0} b_{0}^{2}+b_{0}^{3}\right)\right\},
\end{aligned}
$$

$$
\begin{aligned}
I(y, z)= & A_{i}\left\{\frac{y}{\omega_{0}}-\left[\frac{z^{2}}{4 k \omega_{0}^{4}}-\left(4 a_{0} b_{0}+b_{0}^{2}\right)\right]+\frac{z\left(a_{0}+b_{0}\right)}{k \omega_{0}^{2}} i\right\} \times \\
& A_{i}\left\{\frac{y}{\omega_{0}}-\left[\frac{z^{2}}{4 k \omega_{0}^{4}}-\left(4 a_{0} b_{0}+b_{0}^{2}\right)\right]-\frac{z\left(a_{0}+b_{0}\right)}{k \omega_{0}^{2}} i\right\} \times \\
& \exp \left\{2\left(a_{0}+b_{0}\right)\left\{\frac{y}{\omega_{0}}-\left[\frac{z^{2}}{2 k^{2} \omega_{0}^{4}}-\left(4 a_{0} b_{0}+b_{0}^{2}\right)\right]\right\}\right. \\
& +\left(\left(3 a_{0} b_{0}^{2}+b_{0}^{3}\right)\right\},
\end{aligned}
$$

with

$$
\begin{aligned}
& b_{0}=\frac{z^{2} c}{k^{2} \omega_{0}^{2}}, \\
& c_{0}=\frac{1}{2 \sigma^{2}}+\frac{1}{\rho_{0}^{2}} .
\end{aligned}
$$

Other optical field intensity values in equation (9) can be achieved through changing the plus or minus signs of the transversal coordinates $x$ and $y$ in equations (10) and (11). Each beamlet of the PCAFABs in atmospheric turbulence follows a ballistic trajectory in the $x-z$ and $y-z$ planes; for example, the central beam location with the changes $z$ in the first quadrant is described by the following parabolas:

$$
x=y=\left[\frac{z^{2}}{4 k \omega_{0}^{4}}-\left(4 a_{0} b_{0}+b_{0}^{2}\right)\right] \times \omega_{0} .
$$

Other three beamlets' trajectory coordinates can be acquired by changing the plus or minus signs of the transversal coordinates $x$ and $y$ in equation (13).

\section{Graphical Results and Analysis}

In this section, as numerical examples, we investigate the influence of beam parameters and turbulence factors on the PCAFABs' evolution characteristics at different cross sections of atmospheric environment. Firstly, the variations of beam intensity at source plane and beam transverse acceleration parabolic trajectory with $a_{0}$ are demonstrated in Figure 1, and other parameters are listed as follows: $\lambda=1.55 \mathrm{um}, \sigma=100 \mathrm{~mm}, \omega_{0}=0.2 \mathrm{~m}$, and $C_{n}{ }^{2}=10^{-11} \mathrm{~m}^{-2 / 3}$. It shows that the side lobes of incident PCAFABs gradually diminish with the increasing $a_{0}$, and the beam evolves into a partially coherent array Gaussian beams (PCAGBs) when $a_{0}=0.5$, as learned in Figure 1(c). Moreover, one can observe from Figure 1(d) that three lines of beam transverse acceleration parabolic trajectory overlap each other, manifesting that the central main lobe location is rarely affected by the changes of $a_{0}$. 


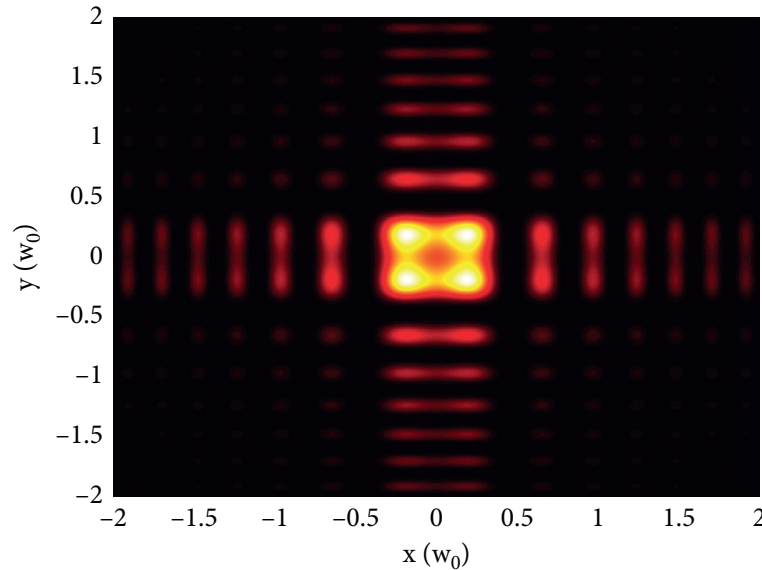

(a)

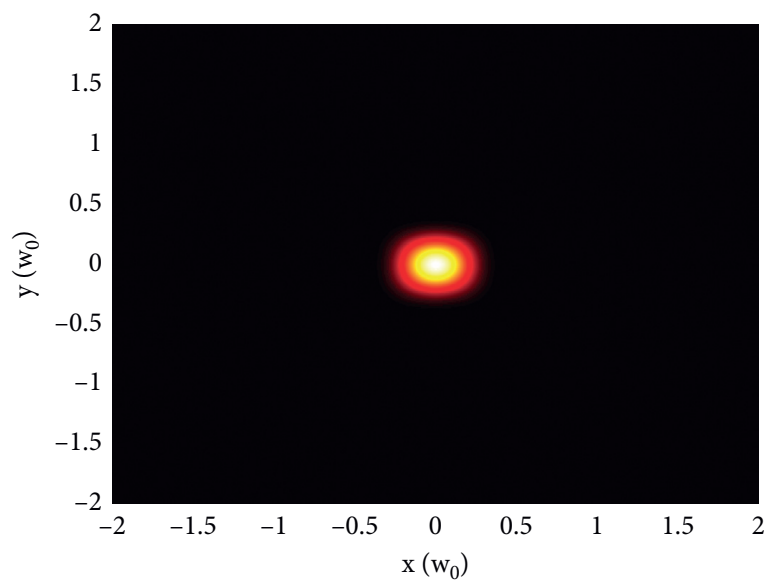

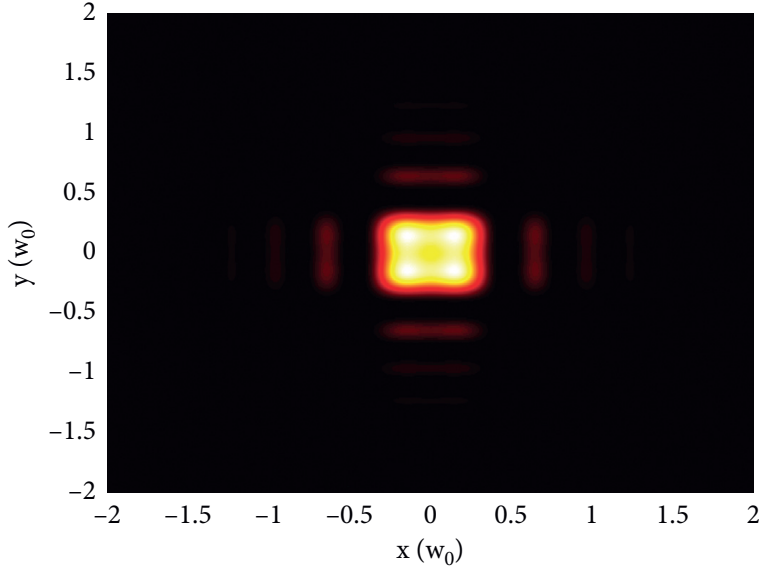

(b)

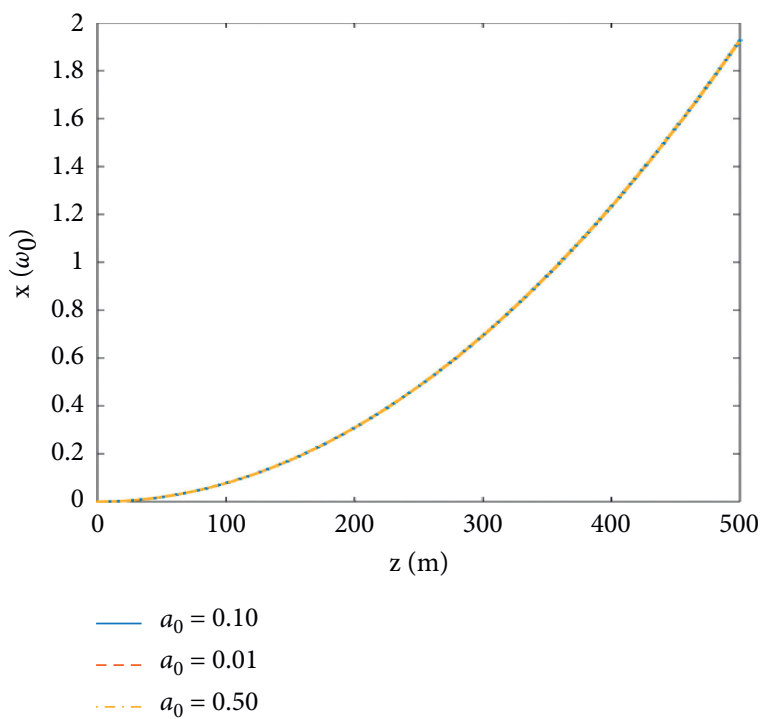

(d)

Figure 1: Influence of $a_{0}$ on PCAFABs' evolution characteristics; other parameters are fixed as $\lambda=1.55 \mathrm{um}, \sigma=100 \mathrm{~mm}, \omega_{0}=0.2 \mathrm{~m}$, and $C_{n}{ }^{2}=10^{-13} \mathrm{~m}^{-2 / 3}$ : (a) incident PCAFABs, $a_{0}=0.01$; (b) incident PCAFABs, $a_{0}=0.1$; (c) incident PCAFABs, $a_{0}=0.5$; (d) beam transverse acceleration parabolic trajectory for three $a_{0}$.

The influence of $\sigma$ on incident PCAFABs' silhouette is depicted in Figure 2, and other parameters are the same as those in Figure 1 except for $a_{0}=0.1$ fixed. It is gotten that the initial coherence length plays an insignificant role on incident beam intensity distribution by comparing Figure 2(a) and Figure 2(b). As the transverse distance increases, the exponential term

$$
\exp \left(a_{0} \frac{r_{1}^{\prime}+r_{2}^{\prime}}{\omega_{0}}\right) \gg \exp \left[-\frac{\left(r_{1}^{\prime}-r_{2}^{\prime}\right)^{2}}{\sigma^{2}}\right]
$$

Thus, two drawings below are much of a muchness.

In order to better understand the effect of the coherence length on beam's evolution characteristics, Figure 3 describes the contour graphs of PCAFABs with different $\sigma$ at several intersecting surfaces during evolution in atmospheric turbulence, and other parameters are the same as those in Figure 1 except for $a_{0}=0$ fixed. It is recognized from these figures that the side loses of PCAFABs fade away with the $\sigma$ decreases and the transmission distance increases. There are great deals of side lobs spreading over each coordinate axis when $\sigma=1 \mathrm{~mm}$ and $z=100 \mathrm{~m}$, as shown in Figure 3(c); however, by viewing Figure 3(d), we discover that lots of these side lobes disappear when beam transmits to $z=500 \mathrm{~m}$; hence, we elucidate that the larger the $\sigma$ can result in the stronger antiturbulence ability of the PCAFABs when it propagates in atmospheric environment. Meanwhile, we find that the beam's self-bending property is increasingly obvious along with the changing of $\sigma$ during the wave transmission process by viewing in Figure 3(f) and Figure $3(\mathrm{~g})$, but these lines are nonmonotonic versus the increases of distance $z$. A mass of simulations manifest that the PCAFABs covert into the PCAGBs at the first maximum point (inflexion A); hence, equation (9) is no longer valid to describe the intensity of PCAFABs when the transmission distance $z$ is larger than the inflexion $\mathrm{A}$. Observed in 


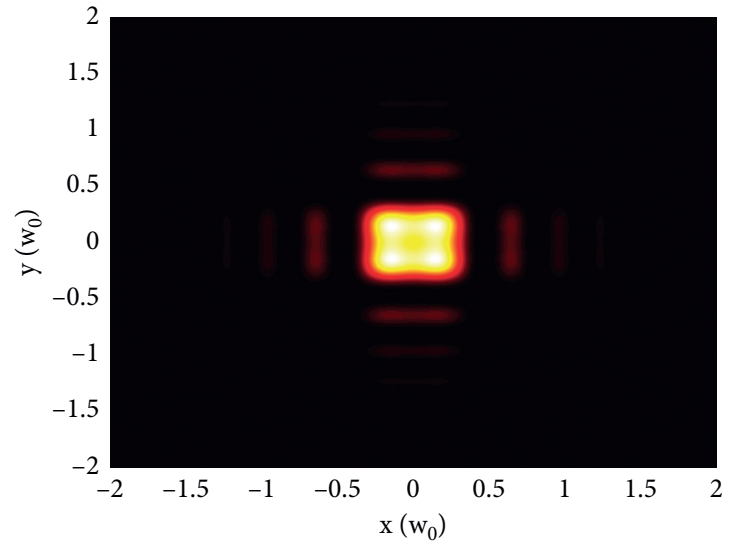

(a)

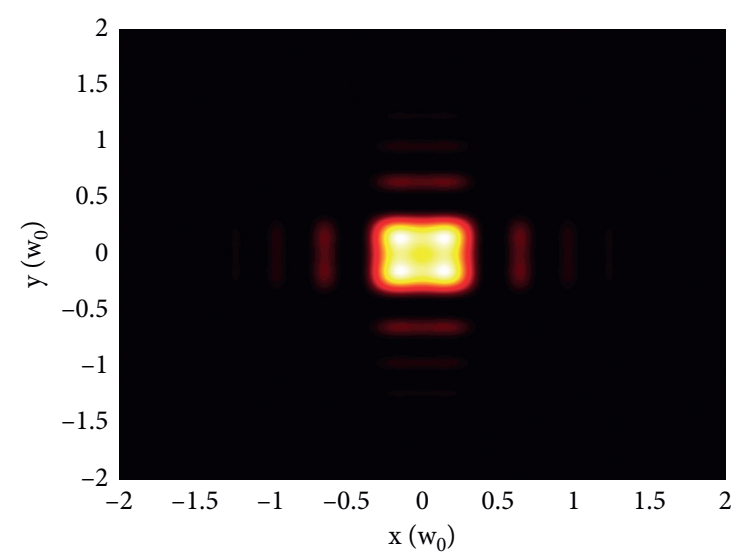

(b)

Figure 2: Incident PCAFABs changed with different $\sigma$; other parameters are the same as those in Figure 1 except for $a_{0}=0.1$ : (a) $\sigma=0.5 \mathrm{~mm}$; (b) $\sigma \longrightarrow \infty$.

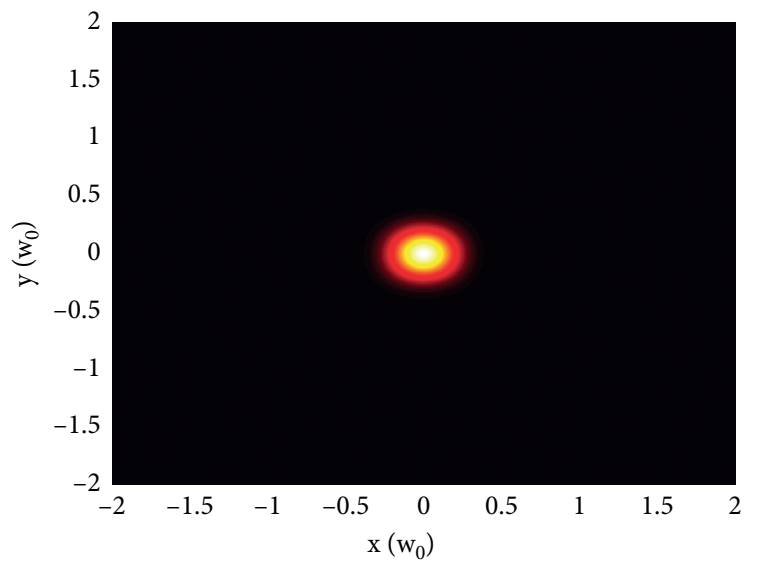

(a)

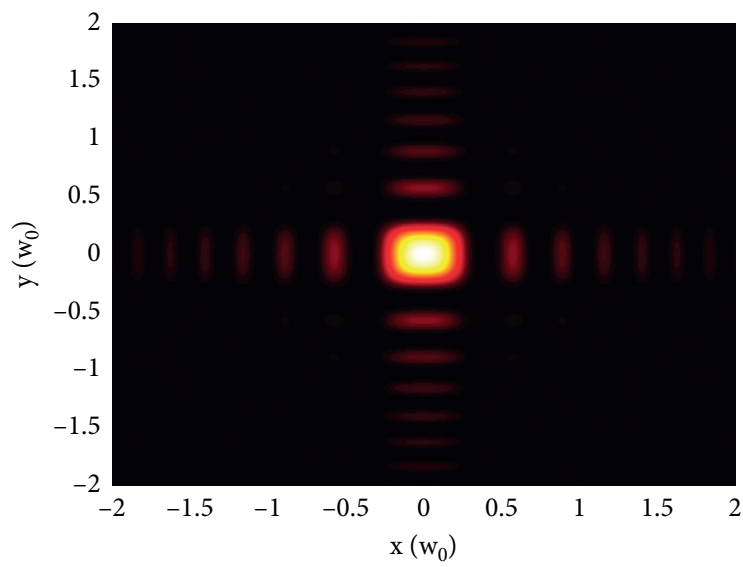

(c)

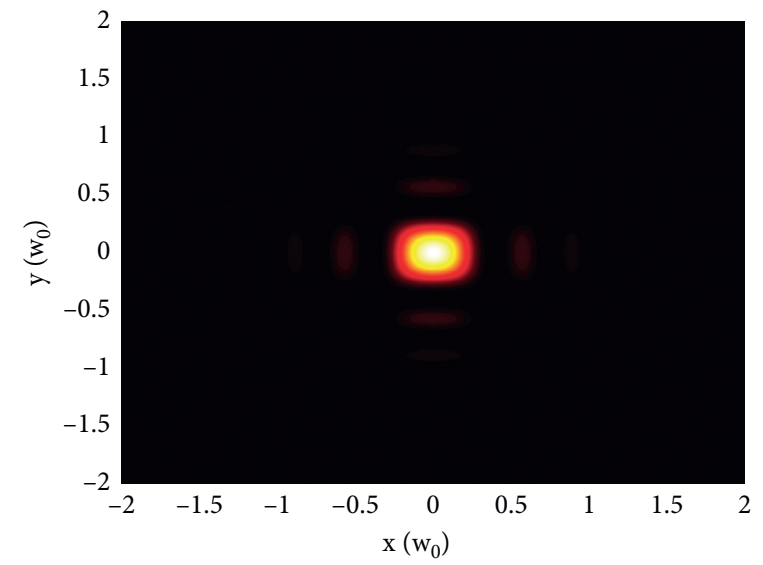

(b)

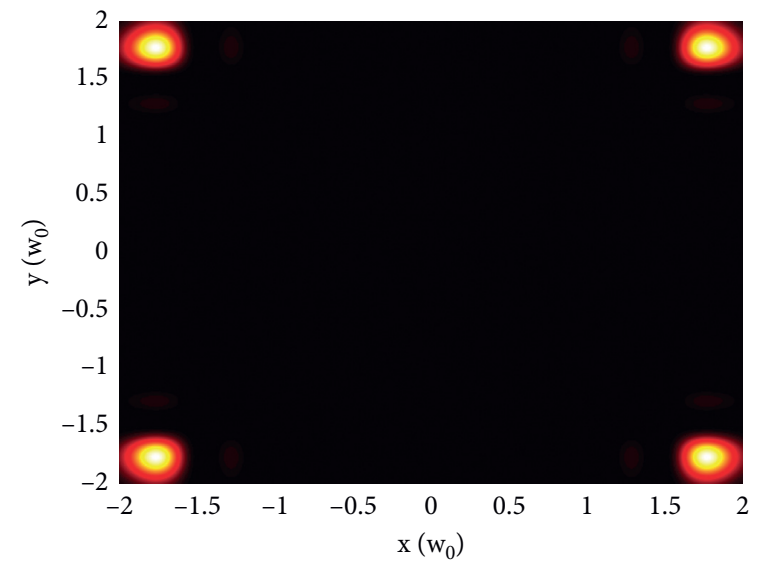

(d)

Figure 3: Continued. 


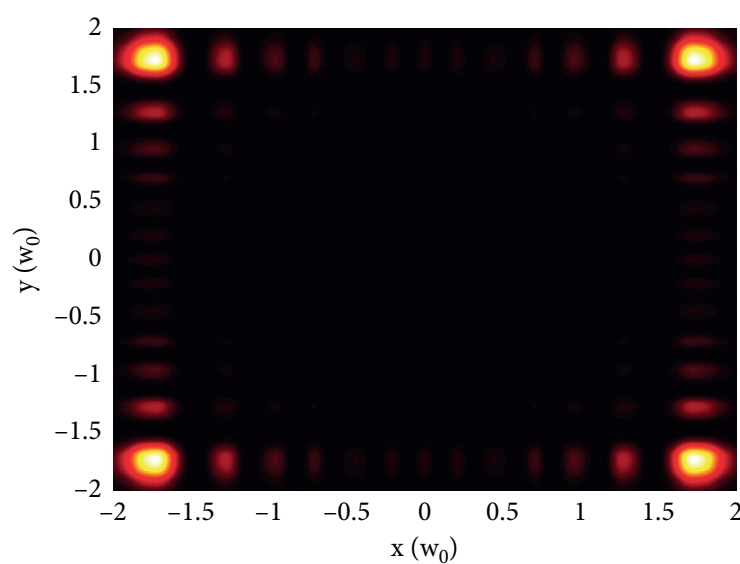

(e)

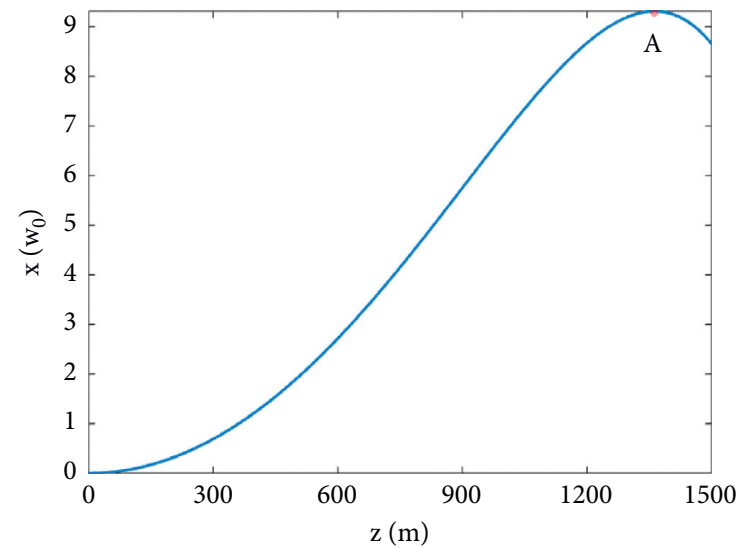

(g)

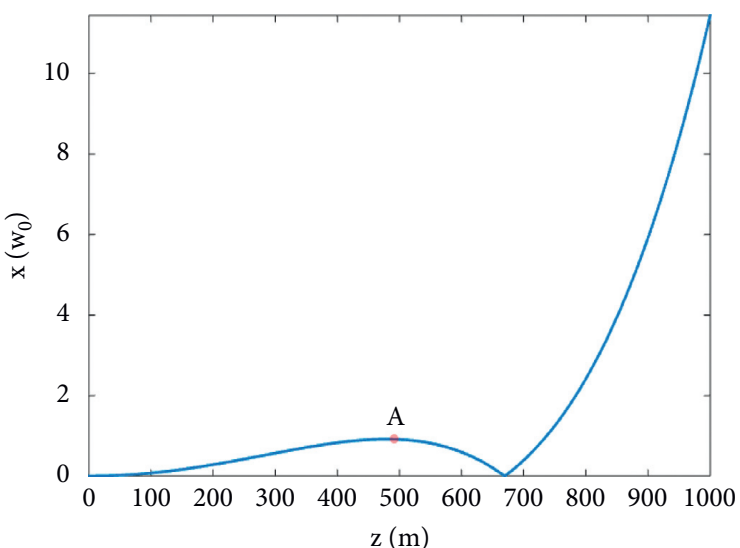

(f)

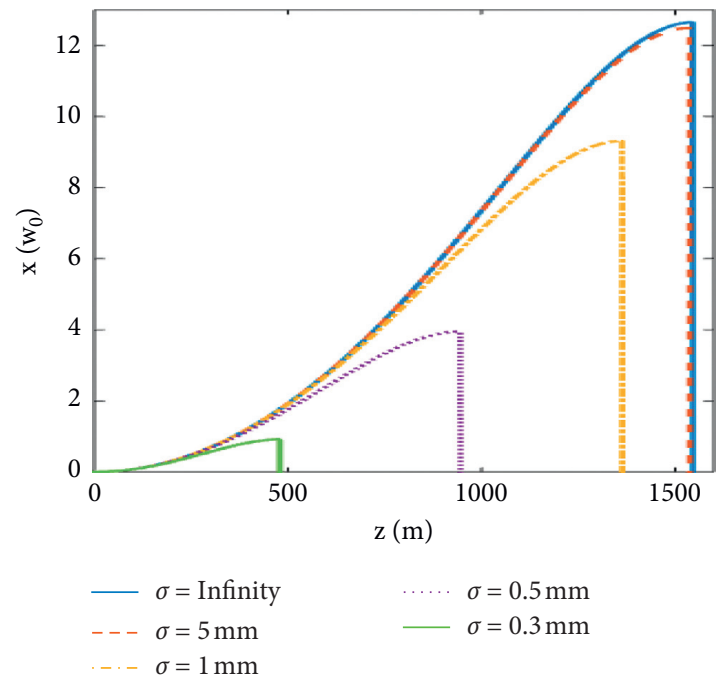

(h)

Figure 3: Influence of $\sigma$ on PCAFABs' evolution characteristics; A expresses the inflexion point, and other parameters are the same as those in Figure 1 except for $a_{0}=0$ : (a) $\sigma=0.1 \mathrm{~mm},(\mathrm{z})=100 \mathrm{~m}$; (b) $\sigma=0.3 \mathrm{~mm}, z=100 \mathrm{~m}$; (c) $\sigma=1 \mathrm{~mm}, z=100 \mathrm{~m}$; (d) $\sigma=1 \mathrm{~mm}, z=500 \mathrm{~m}$; (e) $\sigma=10 \mathrm{~mm}, z=500 \mathrm{~m}$; (f) parabolic trajectory for $\sigma=0.3 \mathrm{~mm}$; (g) parabolic trajectory for $\sigma=1 \mathrm{~mm}$; (h) beam transverse self-acceleration parabolic trajectory for different $\sigma$.

Figure $3(\mathrm{~h})$, it is indicated that the bigger the $\sigma$ is, the more severe the bending is formed at the receiver plane, and the inflexion point $\mathrm{A}$ increases with the increasing of $\sigma$. As we know, the PCAFABs convert into a AFABs when $\sigma \longrightarrow \infty$ and the beam self-bending acceleration at this point is the fastest than that of the other $\sigma$. However, on the other hand, the PCAFABs can result in better directive performance than that of the AFABs while transmission in the same atmospheric circumstances. Hence, we can regulate the path of the PCAFABs in atmospheric environment by choosing the different value of the $\sigma$ to meet tangible needs.

Next, we delve into the effect of $C_{n}{ }^{2}$ on the PCAFABs' deviation at some observation planes, the results are delineated in Figure 4, and we set other parameters to be the same as those in Figure 1(b). The acceleration direction of each beamlet is labeled by the blue arrow head, as shown in Figure 4(a). By viewing the beam intensity evolution rules at the circumstances $C_{n}{ }^{2}=10^{-13} \mathrm{~m}^{-2 / 3}$ in Figure $4(\mathrm{~b})$ and
Figure 4(c), it is found that each beamlet of the PCFAABs gradually converges to the original central location at the beginning of the propagation process until the smallest main and side spots are formed. Then, they deviate farther and farther away from the original central location with the increasing distance $z$ in the rest of atmospheric turbulence. When $z=500 \mathrm{~m}$ in Figure 4(c), the PCAFABs separate into four independent portions because of the self-bending peculiarity, which confirms the conclusion obtained in [22]. Moreover, we find that the side lobes of PCAFABs gradually fade away during transmission in atmospheric environment, and the larger the $C_{n}{ }^{2}$ is, the shorter the transmission distance $z$ is required to achieve the disappearance of beam side petals. While $C_{n}{ }^{2}=10^{-11} \mathrm{~m}^{-2 / 3}$, the transmission distance is $z=500$ where the PCAFABs gradually degrade into the PCAGBs, as can be seen in Figure 4(f). The singular properties of the PCAFABs cease to exist provided that its side lobes disappear entirely; therefore, while $C_{n}{ }^{2}=10^{-8} \mathrm{~m}^{-2 /}$ 


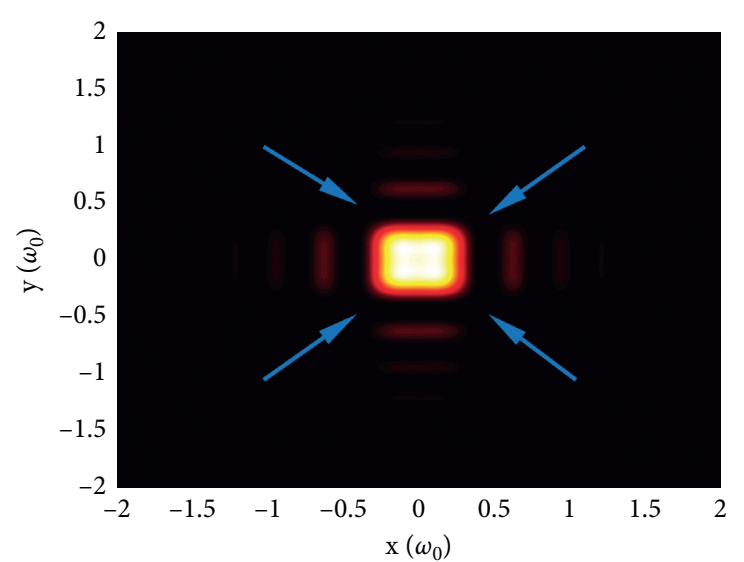

(a)

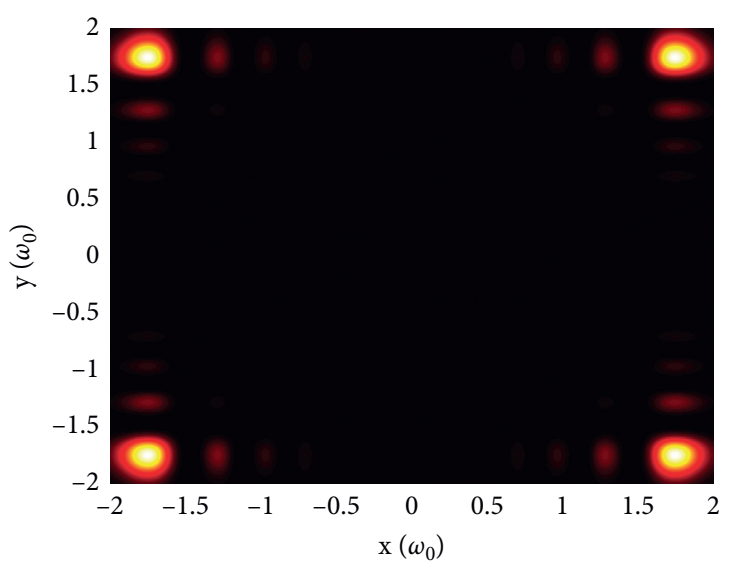

(c)

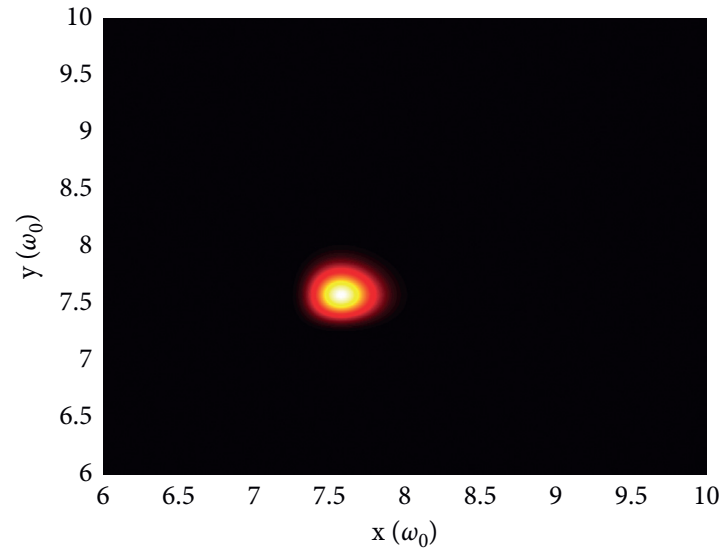

(e)

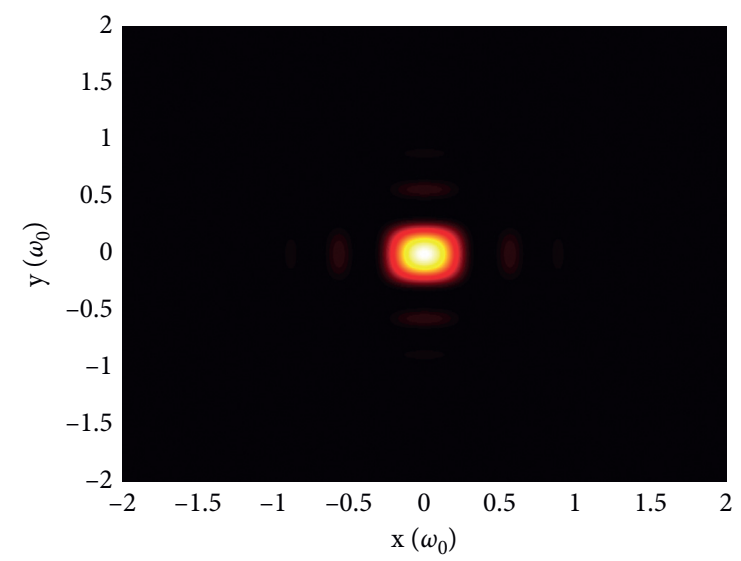

(b)

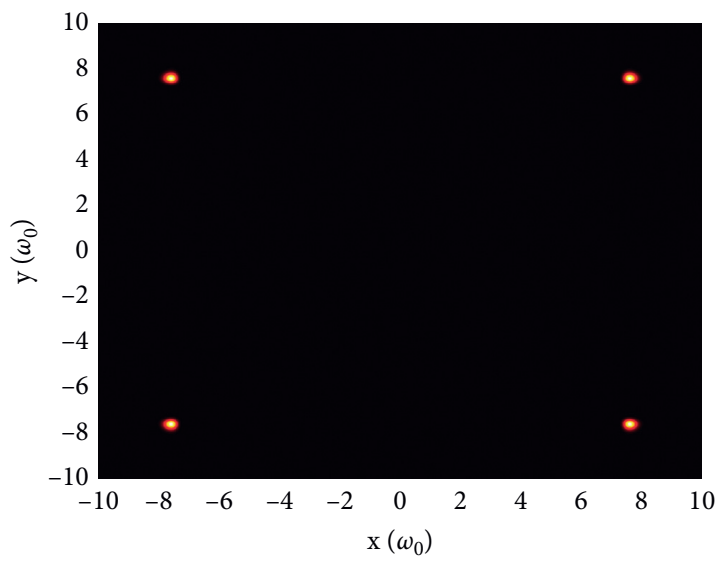

(d)

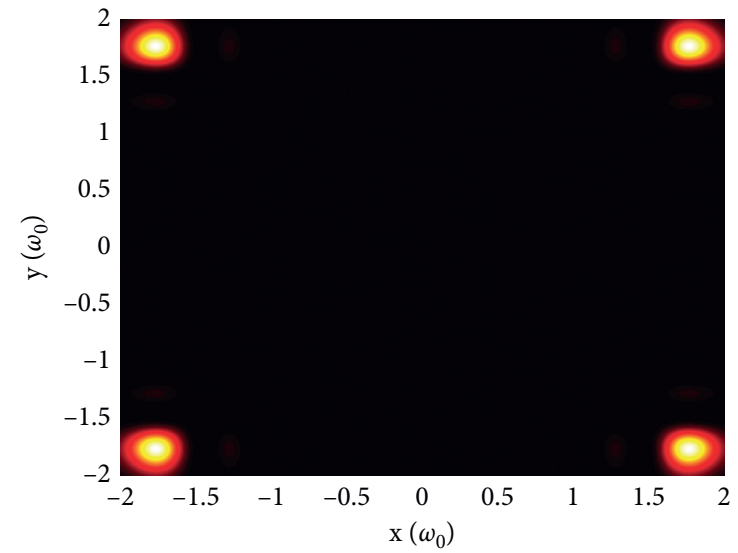

(f)

Figure 4: Continued. 


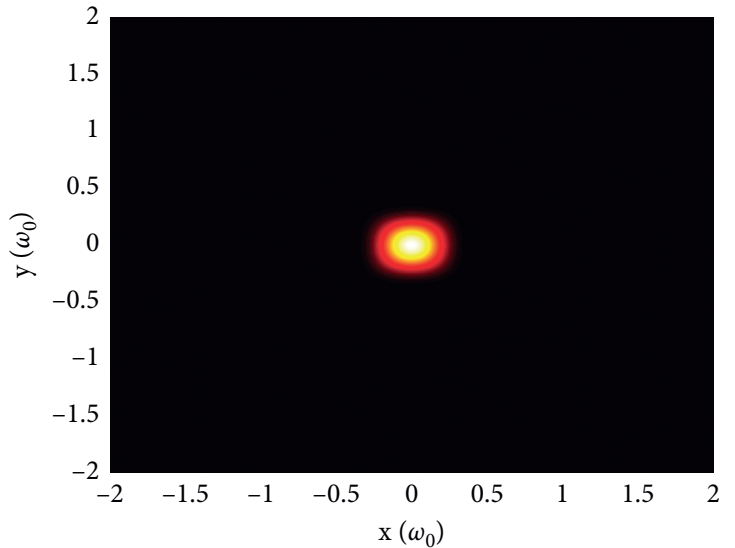

(g)

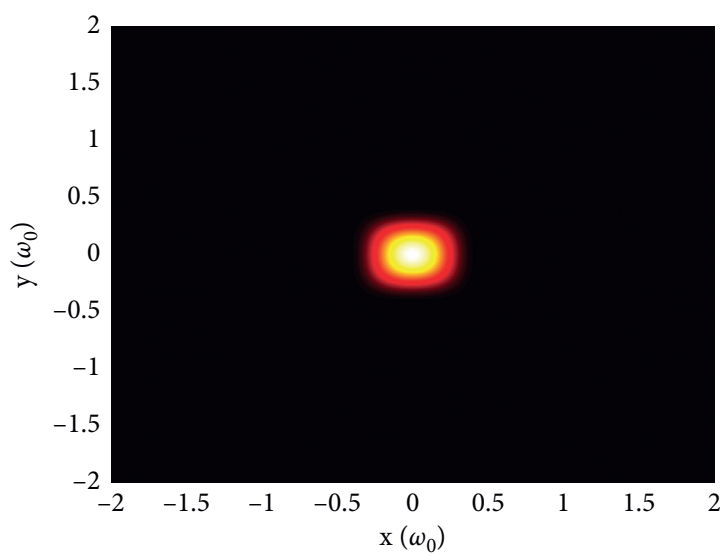

(i)

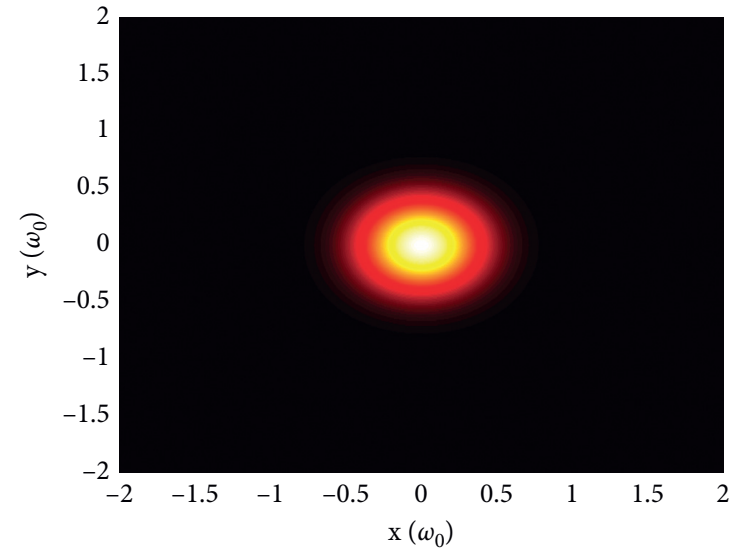

(h)

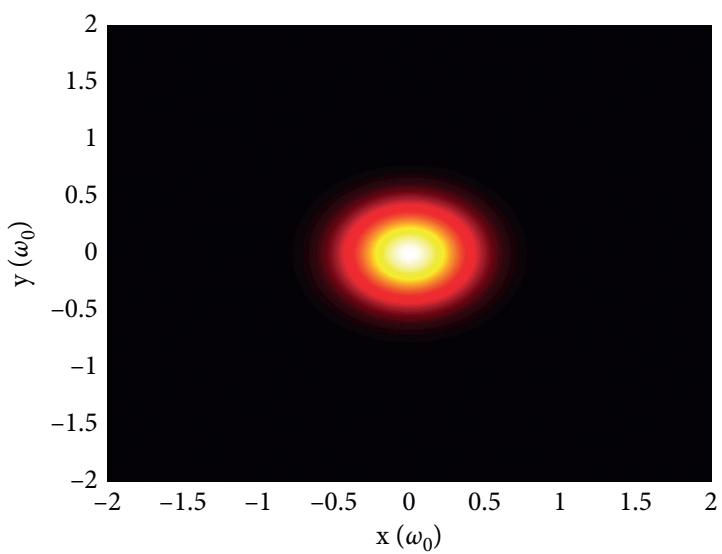

(j)

FIGURE 4: Influence of $C_{n}{ }^{2}$ on PCAFAB evolution characteristics; other parameters are the same as those in Figure 1(b), and the blue arrow head denotes each beamlet's acceleration direction: (a) $C_{n}{ }^{2}=10^{-13} \mathrm{~m}^{-2 / 3},(\mathrm{z})=50 \mathrm{~m} ;(\mathrm{b}) C_{n}{ }^{2}=10^{-13} \mathrm{~m}^{-2 / 3},(\mathrm{z})=100 \mathrm{~m} ;(\mathrm{c}) C_{n}^{2}=10^{-13} \mathrm{~m}^{-2 / 3}$, $(\mathrm{z})=500 \mathrm{~m} ;(\mathrm{d}) C_{n}{ }^{2}=5 \times 10^{-12} \mathrm{~m}^{-2 / 3},(\mathrm{z})=1000 \mathrm{~m}$; (e) zoom in on Figure $4(\mathrm{~d})$ in the first quadrant; (f) $C_{n}^{2}=10^{-11} \mathrm{~m}^{-2 / 3},(\mathrm{z})=500 \mathrm{~m}$; (g) $C_{n}^{2}=10^{-9} \mathrm{~m}^{-2 / 3},(\mathrm{z})=100 \mathrm{~m}$; (h) $C_{n}^{2}=10^{-9} \mathrm{~m}^{-2 / 3},(\mathrm{z})=250 \mathrm{~m} ;(\mathrm{i}) C_{n}^{2}=10^{-8} \mathrm{~m}^{-2 / 3},(\mathrm{z})=50 \mathrm{~m} ;(\mathrm{j}) C_{n}^{2}=10^{-8} \mathrm{~m}^{-2 / 3},(\mathrm{z})=100 \mathrm{~m}$.

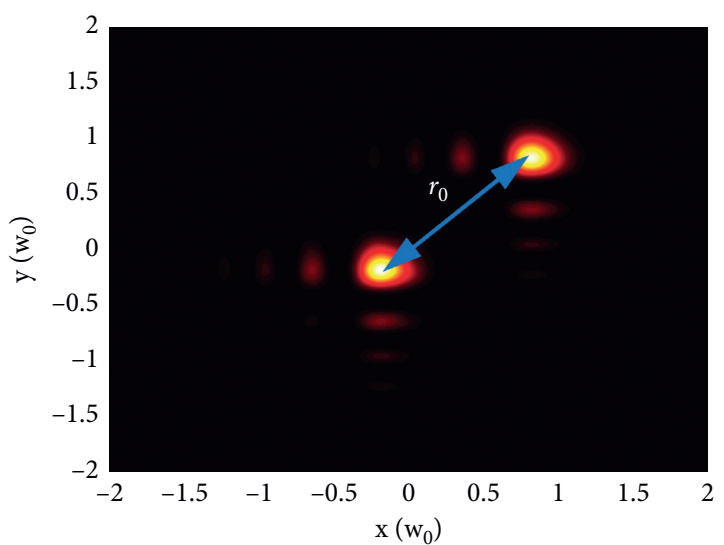

(a)

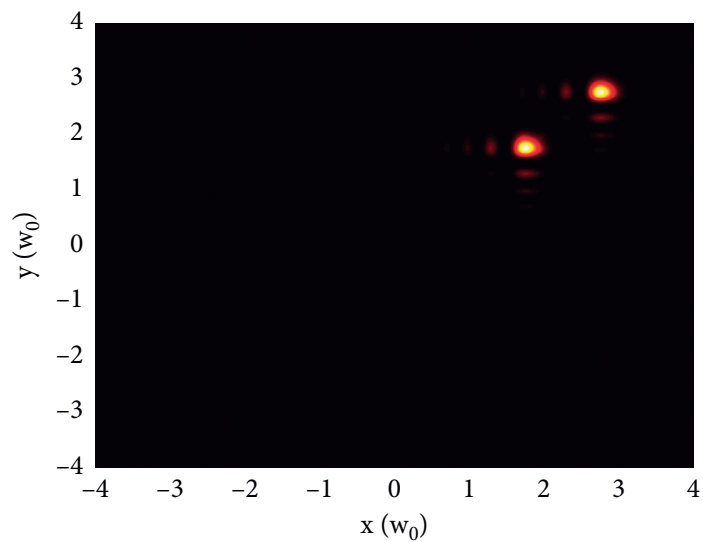

(b)

Figure 5: Continued. 


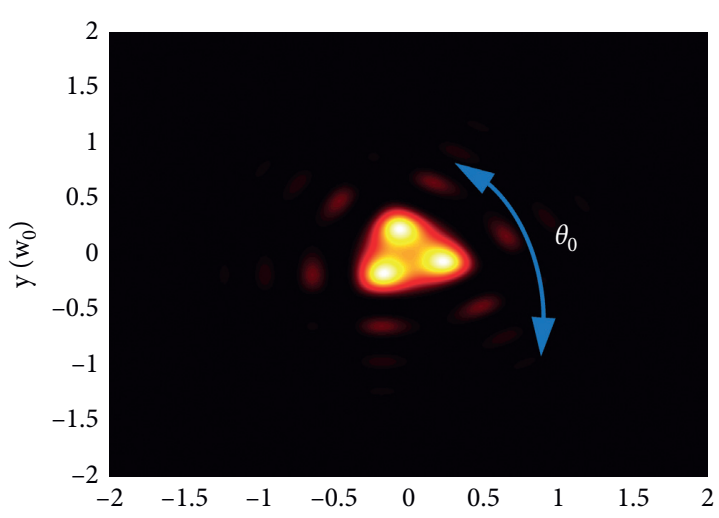

(c)

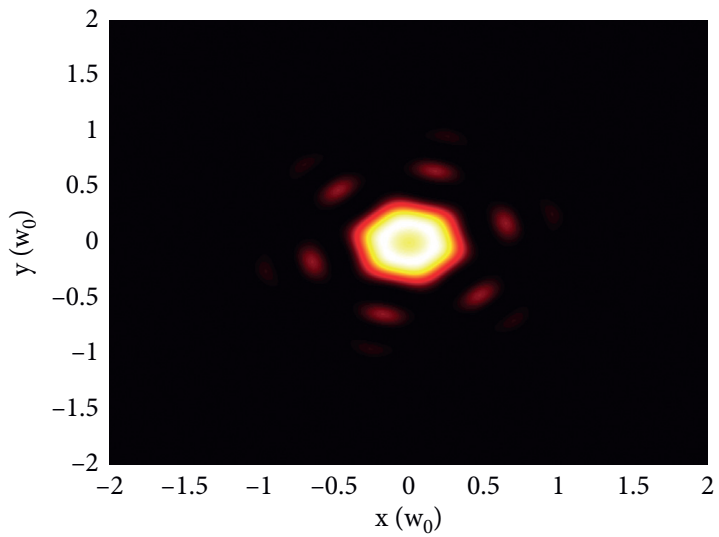

(e)

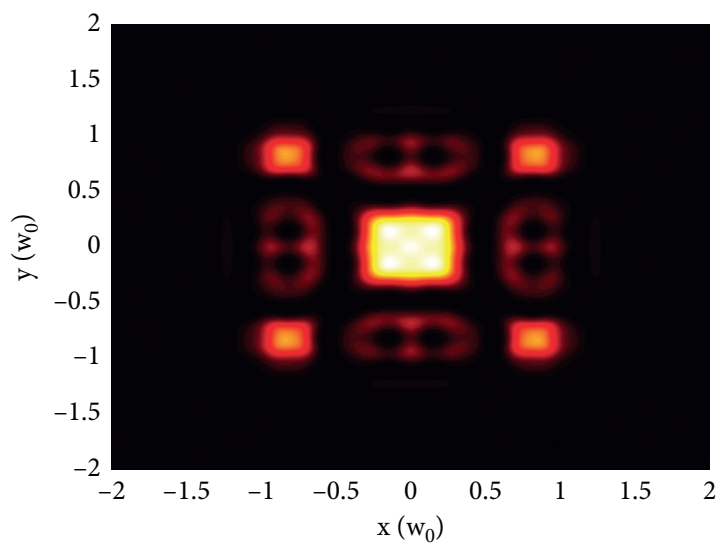

(g)

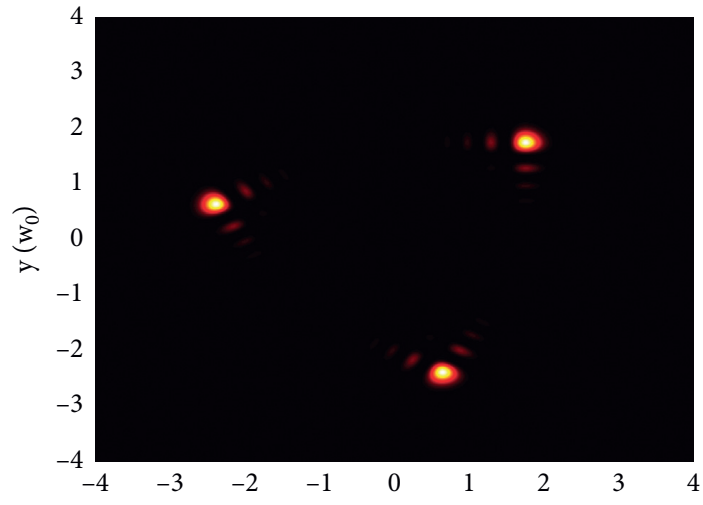

(d)

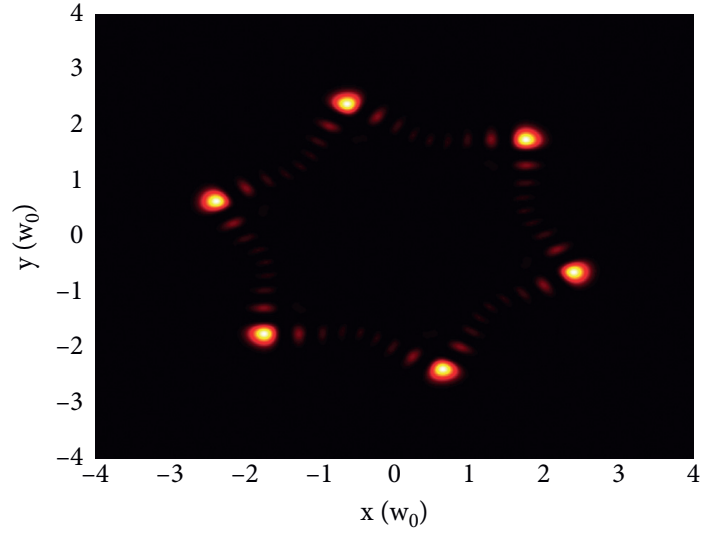

(f)

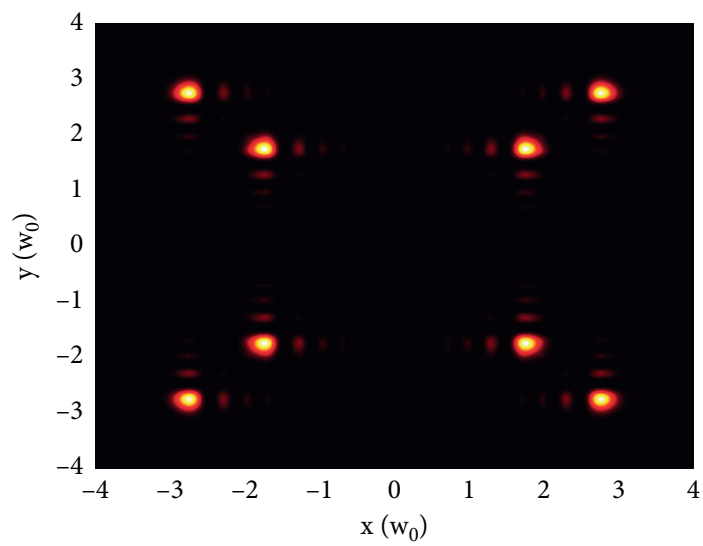

(h)

FIgUre 5: Continued. 


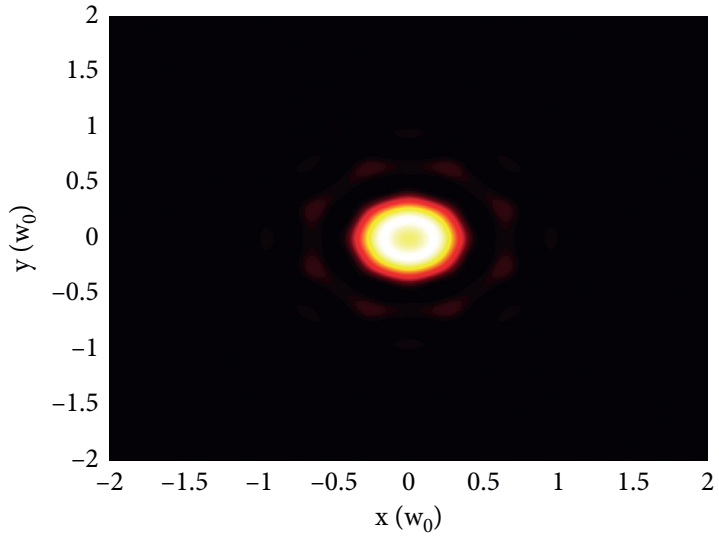

(i)

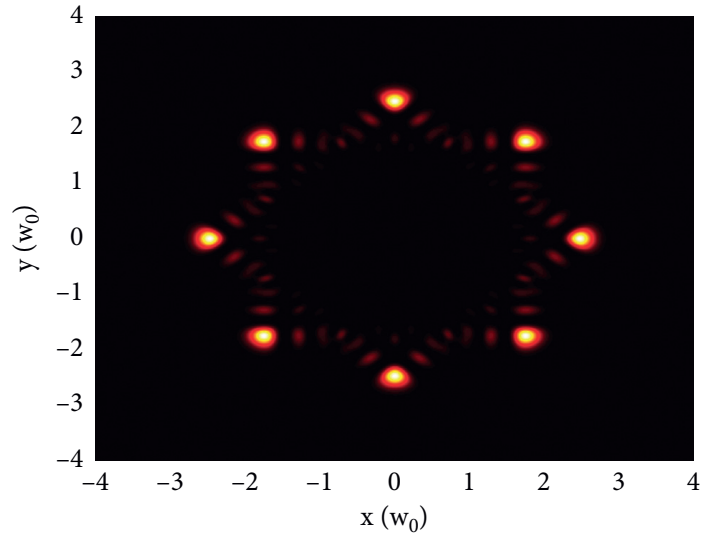

(j)

Figure 5: Transverse intensity distribution of PCAFABs in atmospheric space for different value of $(n)$; other parameters are the same as those in Figure 4(a), and two blue arrow heads denote the initial transverse displacement and angle displacement between two incoming Airy beams, respectively: (a) $(n)=2,(z)=0 \mathrm{~m}$; (b) $(n)=2,(z)=500 \mathrm{~m} ;(\mathrm{c})(n)=3,(z)=0 \mathrm{~m} ;(\mathrm{d})(n)=3,(z)=500 \mathrm{~m} ;(\mathrm{e})(n)=6,(z)=0 \mathrm{~m}$; (f) $(n)=6,(z)=500 \mathrm{~m} ;(\mathrm{g})(n)=8,(z)=0 \mathrm{~m}$, first kind; (h) $(n)=8,(z)=500 \mathrm{~m}$, first kind; (i) $(n)=8,(z)=0 \mathrm{~m}$, second kind; (j) $(n)=8$, $(z)=$ $500 \mathrm{~m}$, second kind.

3 in Figure 4(j), the PCAFABs will never separate from each other in the rest of the atmospheric turbulence, and the spot size of these array beams increases in proportion to the Gaussian function with the increasing transmission distance until the power eventually burns out.

We end up with this section by discussing the PCAFABs through atmospheric turbulence which contains different numbers of the single Airy beam so that the conclusions obtained are universal. The results are presented in Figure 5, where $r_{0}$ and $\theta_{0}$ are the initial transverse displacement and angle displacement between two incoming Airy beams, respectively, $n$ denotes the beam number, and other parameters are in accord with that in Figure 4(a). It reveals from these figures that the incident PCAFAB intensity profile is determined by the $a_{0}, \omega_{0}, r_{0}, \theta_{0}$, and $n$, whereas the beam transmission properties in atmospheric space are characterized by the $C_{n}{ }^{2}, \sigma$, and $z$; in other words, the derived analytical formulae and conclusions can be extended to the study on different numbers of array Airy beams' transmission in atmospheric turbulence.

\section{Conclusion}

In this paper, we have explored thoroughly the properties of the PCAFABs evoluting in the Kolmogorov atmospheric turbulence based on the extended Huygens-Fresnel integral and the Rytov phase structure function under the paraxial approximation. The influence of beam parameters and turbulence factors on the PCAFABs normalized intensity distribution at different cross sections of atmospheric environment was discussed in detail. We found that the side lobes of incident PCAFABs gradually diminish with the increasing $a_{0}$, but the central main lobe location is rarely affected by the changes of $a_{0}$. In stark contrast, $\sigma$ plays an insignificant role on the incident PCAFABs' intensity distribution, but the side loses of
PCAFABs fade away during the transmission process with the $\sigma$ decreases, and the beam self-bending property is increasingly obvious along with the changing of $\sigma$; the bigger the $\sigma$ is, the more severe the bending is formed at the receiver plane, but these lines are nonmonotonic versus the increases of distance $z$. A mass of simulations manifest that the PCAFABs covert into the PCAGBs at the first inflexion; hence, equation (9) is no longer valid to describe the intensity of PCAFABs when the transmission distance $z$ is larger than the inflexion A. Moreover, it is found that the side lobes of PCAFABs gradually diminish during transmission in atmospheric space, and the larger the $C_{n}{ }^{2}$ is, the shorter the transmission distance $z$ is required to achieve complete disappearance of the side petals. We look forward to that the derived analytical formulae and the corresponding conclusions can be useful for learning the optical properties of the PCAFABs which contains different numbers of single Airy beam transmitting in the atmospheric turbulence, especially for free-space optical communication area.

\section{Data Availability}

The data used to support the findings of this study are available from the corresponding author upon request.

\section{Conflicts of Interest}

The authors declare that there are no conflicts of interest regarding the publication of this paper.

\section{Acknowledgments}

The authors gratefully acknowledge the financial support from the Hubei Provincial Department of Education in China (no. D20201803). 


\section{References}

[1] M. N. O. Sadiku, S. M. Musa, and S. R. Nelatury, "Free space optical communications: an overview," European Scientific Journal, vol. 12, no. 9, pp. 55-68, 2016.

[2] X. Chu, S. Zhao, Z. Cheng, Y. Li, R. Li, and Y. Fang, "Research progress of Airy beam and feasibility analysis for its application in FSO system," Chinese Science Bulletin, vol. 61, no. 17, Chinese, 1974.

[3] F. Fu and B. Zhang, "The influence of high-frequency phase distortion on the phase correction effect in atmospheric turbulence," Optik, vol. 125, no. 1, pp. 360-365, 2014.

[4] G. Siviloglou, J. Broky, A. Dogariu, and D. Christodoulides, "Observation of accelerating Airy beams," Physical Review Letters, vol. 99, no. 21, Article ID 213901, 2007.

[5] X. Chu, "Evolution of an Airy beam in turbulence," Optics Letters, vol. 36, no. 14, pp. 2701-2703, 2011.

[6] X. Ji, H. T. Eyyuboğlu, G. Ji, and X. Jia, "Propagation of an Airy beam through the atmosphere," Optics Express, vol. 21, no. 2, pp. 2154-2164, 2013.

[7] H. Eyyuboglu and E. Sermutlu, "Partially coherent Airy beam and its propagation in turbulent media," Applied Physics B, vol. 110, no. 4, pp. 451-457, 2013.

[8] A. Yang and Q. Lin, "Polarization characteristics of partially Airy beams propagating in atmospheric turbulence," Acta Physica Sinica, vol. 63, no. 20, pp. 185-191, 2014, Chinese.

[9] Y. Li, L. Wang, and Q. Wang, "Intensity and phase characteristics of ring airy-Gaussian vortex beam in atmospheric turbulence," Laser and Optoelectronics Progress, vol. 56, no. 14, pp. 19-26, 2019, Chinese.

[10] A. Belafhal, L. Ez-Zariy, S. Hennani, and H. Nebdi, "Theoretical introduction and generation method of a novel nondiffracting waves: olver beams," Optics and Photonics Journal, vol. 05, no. 07, pp. 234-246, 2015.

[11] L. Wang, X. Ji, X. Li, T. Wang, H. Yu, and Q. Li, "Focusing and self-healing characteristics of Airy array beams propagating in self-focusing media," Applied Physics B, vol. 125, no. 9, p. 165, Article ID 165, 2019.

[12] Y. Gu and G. Gbur, "Scintillation of Airy beam arrays in atmospheric turbulence," Optics Letters, vol. 35, no. 20, pp. 3456-3458, 2010.

[13] C. Chen, H. Yang, M. Kavehrad, and Z. Zhou, "Propagation of radial Airy array beams through atmospheric turbulence," Optics and Lasers in Engineering, vol. 52, pp. 106-114, 2014.

[14] Q. Lu, S. Gao, L. Sheng, J. Wu, and Y. Qiao, "Generation of coherent and incoherent Airy beam arrays and experimental comparisons of their scintillation characteristics in atmospheric turbulence," Applied Optics, vol. 56, no. 13, pp. 3750-3757, 2017.

[15] R. Suarez, A. Neves, and M. Gesualdi, "Generation and characterization of an array of Airy-vortex beams," Optics Communications, vol. 458, no. 1, Article ID 124846, 2020.

[16] L. Jin and X. Zhang, "Propagation properties of airy beam through periodic slab system with negative index materials," International Journal of Optics, vol. 2018, Article ID 9478483, 7 pages, 2018.

[17] V. Varlamov, "Differential and integral relations involving fractional derivatives of Airy functions and applications," Journal of Mathematical Analysis and Applications, vol. 348, no. 1, pp. 101-115, 2008.

[18] P. Wu, X. Ke, and Q. Song, "Realization of experiment on auto-focusing array airy beam," Chinese Journal of Lasers, vol. 45, no. 6, Chinese, Article ID 0605002, 2018.
[19] W. Wen, Y. Jin, M. Hu et al., "Beam wander of coherent and partially coherent Airy beam arrays in a turbulent atmosphere," Optics Communications, vol. 415, pp. 48-55, 2018.

[20] W. Nelson, J. P. Palastro, C. C. Davis, and P. Sprangle, "Propagation of Bessel and Airy beams through atmospheric turbulence," Journal of the Optical Society of America A, vol. 31, no. 3, pp. 603-609, 2014.

[21] J. C. Ricklin and F. M. Davidson, "Atmospheric turbulence effects on a partially coherent Gaussian beam: implications for free-space laser communication," Journal of the Optical Society of America A, vol. 19, no. 9, pp. 1794-1802, 2002.

[22] L. Jin and Q. Wang, "Mechanism of two-dimensional finite airy Gaussian array beams through curved slab systems," Chinese Journal of Physics, vol. 66, pp. 1-8, 2020. 